\title{
Infections Du Site Opératoire À l'Hôpital National De Zinder, Niger: Aspects Épidémiologiques Et Bactériologiques
}

\author{
Mahamadou Doutchi, \\ Département de médecine, Services de maladies infectieuses, Hôpital \\ National de Zinder, Faculté des Sciences de la Santé, \\ Université de Zinder, Niger \\ Harissou Adamou, \\ Département de chirurgie et spécialités chirurgicales, Hôpital National de \\ Zinder, Faculté des Sciences de la Santé, Université de Zinder, Niger
}

\section{Mahamane Lawali Yahaya,} Lawan Ousmane,

Service de Laboratoire Hôpital National de Zinder, Niger

\section{Ibrahim Amadou Magagi, Maazou Halidou, Moussa Taofik, Mansour Adamou,}

Département de chirurgie et spécialités chirurgicales, Hôpital National de Zinder, Faculté des Sciences de la Santé, Université de Zinder, Niger

\section{Karamba Sylla,}

Service de maladies infectieuses et tropicales de l'Hôpital National Donka, Faculté de Sciences et techniques de la santé, Université Gamal Abdel

Nasser de Conakry, Guinée

Abdoul Salam Ouedraogo,

Université catholique de l'Afrique de l'Ouest,

Unité Universitaire à Bobo-Dioulasso (UUB), Burkina Faso

\section{Résumé}

Objectif : Rapporter les caractéristiques épidémiologiques et bactériologiques des infections du site opératoire (ISO) dans les services de chirurgie de l'hôpital national de Zinder, Niger. Patients et méthodes : Il s'agissait d'une étude transversale qui s'est déroulée sur une période de 5 mois (Mai à Aout 2016) aux services de chirurgie et de laboratoire de l'Hôpital National de Zinder (HNZ) au Niger incluant tous les patients opérés et ayant 
développé une ISO. Résultats : Durant l'étude, 485 patients avaient été opérés et 38 cas d'ISO étaient enregistrés soit une incidence de 7,83\%. L'âge médian était de 31,5 ans (8-78 ans). Le sexe masculin était majoritaire avec un sexratio de 6,6 (33/5). Les urgences chirurgicales représentaient 57,9\% des cas $(\mathrm{n}=22)$. Selon la classification d'Alte Meier, 13 cas $(34,2 \%)$ étaient considérés comme une chirurgie contaminée et 21 cas $(55,3 \%)$ une chirurgie sale. Les ISO étaient superficielles dans $36,8 \%(\mathrm{n}=14)$, profondes dans $42,1 \%(\mathrm{n}=16)$. Sur les 38 prélèvements analysés, 33 cultures étaient positives soit $86,8 \%$. Les entérobactéries représentaient $69,7 \%(\mathrm{n}=23)$, les Cocci à Gram positif 24,2\% $(\mathrm{n}=8)$. Escherichia coli était l'espèce prédominante avec $45,5 \%(\mathrm{n}=15)$, suivie de Staphylococcus aureus avec 18,2\% ( $\mathrm{n}=6)$. La majeure partie des cultures positives provenait du service de chirurgie viscérale 15 cas $(45,5 \%)$ puis de l'urologie et de traumatologie-orthopédie avec respectivement 10 cas (30,3\%) et 8 cas $(24,2 \%)$. La quasi-totalité des entérobactéries était résistante aux antibiotiques usuels (amoxicilline, amoxicilline-acide clavulanique) et plus de $84 \%$ étaient résistantes à la ceftriaxone. En général les bacilles Gram négatifs étaient sensibles à l'imipenème $(100 \%)$, la colistine $(93,8 \%)$, l'aztréonam $(68,7 \%)$ et le chloramphénicol $(47,8 \%)$. Les cocci-Gram positifs étaient sensibles à la vancomycine, au chloramphénicol et à l'érythromycine dans respectivement $100 \% ; 62,5 \%$ et $57,1 \%$. Conclusion : Les infections du site opératoires représentent un problème majeur. La fréquence des ISO rend indispensable la mise en place d'une équipe multidisciplinaire pour la surveillance de celles-ci. Le fort taux de résistance aux antibiotiques usuels doit susciter des actions préventives et systématiser l'examen bactériologique en cas de suppurations postopératoires.

Mots clés : Infection du Site opératoire, Sensibilité, Profil Bactériologique, Niger 


\title{
Surgical Site Infections at Zinder National Hospital, Niger: Epidemiological and Bacteriological Aspects
}

\author{
Mahamadou Doutchi, \\ Département de médecine, Services de maladies infectieuses, Hôpital \\ National de Zinder, Faculté des Sciences de la Santé, \\ Université de Zinder, Niger \\ Harissou Adamou, \\ Département de chirurgie et spécialités chirurgicales, Hôpital National de \\ Zinder, Faculté des Sciences de la Santé, Université de Zinder, Niger

\section{Mahamane Lawali Yahaya, Lawan Ousmane,} \\ Service de Laboratoire Hôpital National de Zinder, Niger

$$
\begin{gathered}
\text { Ibrahim Amadou Magagi, } \\
\text { Maazou Halidou, } \\
\text { Moussa Taofik, } \\
\text { Mansour Adamou, }
\end{gathered}
$$ \\ Département de chirurgie et spécialités chirurgicales, Hôpital National de \\ Zinder, Faculté des Sciences de la Santé, Université de Zinder, Niger
}

\section{Karamba Sylla,}

Service de maladies infectieuses et tropicales de l'Hôpital National Donka, Faculté de Sciences et techniques de la santé, Université Gamal Abdel

Nasser de Conakry, Guinée

\author{
Abdoul Salam Ouedraogo, \\ Université catholique de l'Afrique de l'Ouest, \\ Unité Universitaire à Bobo-Dioulasso (UUB), Burkina Faso
}

\begin{abstract}
Objective: To report the epidemiological and bacteriological characteristics of surgical site infections (SSI) in the surgical departments of Zinder National Hospital. Patients and methods: This was a cross-sectional study that took place over a period of 5 months (May to August 2016) at the Zinder National Hospital (HNZ) Surgical and Laboratory Services in Niger including all patients operated on and having developed an SSI. Results: During the study, 485 patients had been operated on and 38 cases of ISO were recorded with an incidence of $7.83 \%$. The median age was 31.5 years $(8-78$ years). The male sex was in the majority with a sex ratio of $6.6(33 / 5)$. Surgical
\end{abstract}


emergencies accounted for $57.9 \%$ of cases $(n=22)$. According to Altemeier's classification, 13 cases $(34.2 \%)$ were considered as contaminated surgery and 21 cases $(55.3 \%)$ were dirty surgery. Surgical site infections were superficial in $36.8 \%(n=14)$, deep in $42.1 \%(n=16)$. Of the 38 samplings analyzed, 33 cultures were positive, i.e. $86.8 \%$. The enterobacteria accounted for $69.7 \%$ (n $=23)$, Gram-positive Cocci 24.2\% ( $\mathrm{n}=8)$. Escherichia coli was the predominant species with $45.5 \%(\mathrm{n}=15)$, followed by Staphylococcus aureus with $18.2 \%(n=6)$. The majority of positive cultures came from the visceral surgery department 15 cases $(45.5 \%)$ followed by urology and orthopedic trauma with respectively 10 cases $(30.3 \%)$ and 8 cases $(24.2 \%)$. Virtually all enterobacteria were resistant to the usual antibiotics (amoxicillin, amoxicillinclavulanic acid) and more than $84 \%$ were resistant to ceftriaxone. In general Gram-negative bacilli were sensitive to imipenem (100\%), colistin (93.8\%), aztreonam (68.7\%) and chloramphenicol (47.8\%). Gram-positive cocci were sensitive to vancomycin, chloramphenicol and erythromycin in $100 \%$, respectively; $62.5 \%$ and $57.1 \%$. Conclusion: Surgical site infections represent a major problem. The frequency of SSI makes it essential to set up a multidisciplinary team to monitor them. The high rate of resistance to the usual antibiotics must provoke preventive actions and systematize the bacteriological examination in case of postoperative suppuration.

Keywords: Surgical Site Infection; sensitivity, bacterial profile, Niger

\section{Introduction}

Les infections du site opératoire (ISO) sont des infections nosocomiales survenant au niveau de la plaie opératoire, dans les 30 jours postopératoires ou dans l'année en cas d'implantation de matériel (Fournel 2017; Allegranzi, 2016; Horan, 2008]. Les ISO représentent la 3 ème infection nosocomiale après les infections urinaires et respiratoires [Fournel, 2017]. En dépit du progrès dans les soins chirurgicaux, l'asepsie péri opératoire et de l'antibiothérapie, les ISO restent encore un problème majeur de santé publique du fait du surcoût, du séjour hospitalier long et une morbidité et mortalité élevée (Allegranzi, 2016, Horan, 2008). Les principaux facteurs de risque impliqués dans les ISO sont endogènes (provenant du patient) ou en relation avec l'environnement immédiat pré-, per- et postopératoires (Fournel, 2017; Horan, 2008; Allegranzi, 2016; Baker, 2016; Sisay, 2019; Alfonso-Sanchez, 2017). L'incidence des ISO dans les pays développés varie de $0,7 \%$ à $5 \%$ allant jusqu'à 33\% en cas de chirurgie abdominale (Fournel, 2017; Baker, 2016; Sisay, 2019). Les ISO sont plus fréquentes en Afrique subsaharienne, leur prévalence peut varier de $6 \%$ à plus de $40 \%$ en fonction des cas (Ouédraogo, 2011; Adrien, 2016; Simon, 2007; Ndayisaba, 1992; Ngaroua, 2016; Adamou, 2016). Au Niger, les ISO ont été diversement rapportées dans 
les études sur les pathologies chirurgicales, toutefois il existe peu des données objectives sur le profil bactériologique des ISO (Abdoulaye, 2018). Cette étude qui entre dans le cadre de la lutte contre les infections associées aux soins et de la lutte contre la résistance des bactéries aux antibiotiques vise à rapporter les caractéristiques épidémiologiques et bactériologiques des infections du site opératoire à l'Hôpital National de Zinder, Niger.

\section{Matériels et méthodes}

Il s'agissait d'une étude transversale qui s'est déroulée sur une période de 5 mois (Mai à Septembre 2016) aux services de chirurgie et de laboratoire de l'Hôpital National de Zinder (HNZ) au Niger. Nous avons inclus dans cette étude tous les patients sans distinction de sexe, d'âge, de provenance, opérés et ayant développé une infection du site opératoire dans un délai supérieur ou égal à 48 heures et ayant bénéficié d'un prélèvement bactériologique.

Il s'agissait des patients opérés pour une affection relevant de la chirurgie digestive (CD), de l'urologie (Uro) de la traumato-orthopédie (TO). Ils étaient hospitalisés dans les services suivants : chirurgie générale B (service de chirurgie générale et digestive constitué des salles communes), chirurgie catégorie $\mathrm{C}$ (service des salles uniques qui reçoit les patients de chirurgie générale et digestive, d'urologie et de traumato-orthopédie), urologie (salles communes) et traumato-orthopédie (salles communes). Les patients dont la durée d'hospitalisation est inférieure à 48 heures et ayant une fièvre étaient exclus de l'étude. Le diagnostic d'ISO (survenue dans les 30 jours suivant l'acte chirurgical ou dans l'année si implant profond) était établi sur de critères cliniques et bactériologiques. Les patients étaient répartis en trois catégories (Baker, 2016; Allegranzi, 2016; Carlet, 2015) :

- Infections superficielles du site opératoire qui correspondent à l'atteinte des tissus cutanés ou sous-cutanés. Il existe au moins un de ces signes suivants : pus exonéré de la partie superficielle de l'incision, mise en évidence d'un germe par prélèvement superficiel de l'incision, symptôme d'inflammation (rouge, chaud, douloureux...). Le diagnostic d'ISO superficielles de l'incision est porté par le chirurgien.

- Infections profondes du site opératoire qui correspondent à l'atteinte des tissus profonds ou de l'espace du site opératoire. Elles sont caractérisées par un écoulement purulent par le drain, une déhiscence de la cicatrice associée à une fièvre et de douleur. Le diagnostic d'infection de la partie profonde de l'incision ou de l'espace opératoire est porté par le chirurgien. L'isolement du microorganisme et l'imagerie aident au diagnostic positif de l'infection.

- Infection de l'organe, du site ou de l'espace : Il s'agit d'une infection qui touche l'organe ou l'espace du site opératoire (toute partie anatomique, autre que l'incision, ouverte ou manipulée pendant 
l'intervention), et pour laquelle on constate au moins un des signes suivants : du pus (provenant d'un drain), un germe isolé à partir d'une culture, un abcès ou un autre signe évident d'infection de l'organe ou de l'espace.

Les interventions chirurgicales étaient réparties en 4 stades de contamination selon la classification d'Altemeier [Altemeier et al. 1968] : chirurgie propre, chirurgie propre contaminée, chirurgie contaminée, chirurgie sale.

\section{Technique d'étude au laboratoire}

Les prélèvements étaient effectués soit par ponction à la seringue si les suppurations étaient abondantes soit par écouvillonnage si elles étaient minimes après nettoyage du site opératoire avec de l'eau physiologique stérile. Examen macroscopique: La consistance, la couleur et l'odeur étaient appréciées puis notifiées sur les documents (cahier de paillasse, registre, bulletin).

Examen microscopique: Pour chaque échantillon une observation directe sans coloration était effectuée pour rechercher des éléments cellulaires (leucocytes, hématies, bactéries). Un Examen après coloration : Avec le liquide de suppuration, un premier frottis était réalisé puis coloré au May Grunwald Giemsa (MGG). Par la suite une cytologie quantitative et qualitative des éléments leucocytaires était effectuée à travers une observation microscopique.

Un autre frottis était confectionné et coloré au Gram pour déterminer la morphologie bactérienne et son affinité tinctoriale vis-à-vis du violet de gentiane et de la fuschine. Si la bactérie est colorée en violet, elle est à Gram positif. Par contre si la bactérie est colorée en rose, elle est à Gram négatif. Le résultat de la coloration au Gram permet d'orienter la suite du traitement bactériologique de l'échantillon surtout dans le choix des milieux de culture. Culture : chaque échantillon avait été ensemencé sur une gélose ordinaire (GO), une gélose au sang frais, une gélose chocolat + Polyvitex (GC+ PV), et une gélose Chapman puis incubé à $37^{\circ} \mathrm{C}$ pendant 18 à $24 \mathrm{~h}$. La gélose au sang frais et la gélose chocolat + Polyvitex avaient été incubées en atmosphère aérobie et anaérobie. Un antibiogramme était réalisé en cas de culture positive. Les variables suivantes étaient recueillies : âge, sexe, indications opératoires, délai d'apparition des suppurations, aspects bactériologiques. Les données recueillies ont été saisies sur Excel et analysées avec Epi-info version 3.5.4. Les patients étaient suivis sur une période postopératoire d'au moins 30 jours.

\section{Résultats}

\section{Aspects épidémiologiques}

Durant la période d'étude, 38 patients ont développé une ISO sur un total de 485 patients opérés soit une incidence de 7,83\%. Le sexe masculin 
était majoritaire avec $86,8 \%(n=33)$ soit un sexe-ratio de 6,6. L'âge médian était de 31,5 ans avec des extrêmes de 8 à 78 ans. Les tranches d'âge les plus touchées étaient de 0 à 15 ans pour la chirurgie $\mathrm{B}$, de 16 à 30 ans pour la chirurgie catégorie et le service de trauma-orthopédie, de 61 à 75 ans pour l'urologie. L'âge et la répartition selon le sexe sont décrits dans le tableau I.

L'intervention chirurgicale était faite en urgence dans 57,9\% $(n=22)$ et dans le cadre de la chirurgie programmée dans 42,1\% $(n=16)$. Les interventions chirurgicales étaient classées en quatre groupes selon Altemeier : chirurgie propre 2 cas $(5,3 \%)$, chirurgie propre contaminée 2 cas $(5,3 \%)$, chirurgie contaminée 13 cas $(34,2 \%)$ et chirurgie sale 21 cas $(55,3 \%)$.

Tableau I : Répartition des ISO par sexe et par tranche d'âge selon les services.

\begin{tabular}{|c|c|c|c|c|c|c|c|c|}
\hline \multirow{2}{*}{ Sexe et âge } & \multicolumn{2}{|c|}{ Sexe } & \multicolumn{7}{|c|}{ Tranche d'âge (année) } \\
\cline { 2 - 9 } & $\mathrm{M}$ & $\mathbf{F}$ & $0-15$ & $16-30$ & $31-45$ & $46-60$ & $61-75$ & $76-90$ \\
\cline { 2 - 9 } Services & $\mathrm{N}(\%)$ & $\mathrm{N}(\%)$ & $\mathrm{N}(\%)$ & $\mathrm{N}(\%)$ & $\mathrm{N}(\%)$ & $\mathrm{N}(\%)$ & $\mathrm{N}(\%)$ & $\mathrm{N}(\%)$ \\
\hline Chirurgie B & $11(78,6)$ & $3(21,4)$ & $7(50)$ & $3(21,4)$ & $2(14,3)$ & $2(14,3)$ & 0 & 0 \\
\hline Urologie & $13(66,7)$ & 0 & $1(7,7)$ & 0 & 0 & $3(23,1)$ & $7(53,8)$ & $2(15,4)$ \\
\hline Traumatologie & $5(100)$ & 0 & 0 & $3(60)$ & 0 & $2(40)$ & 0 & 0 \\
\hline Chirurgie C & $4(100)$ & $2(33,3)$ & $1(16,7)$ & $4(66,7)$ & 0 & $1(16,7)$ & 0 & 0 \\
\hline Total & $33(86,8)$ & $5(13,2)$ & $9(23,7)$ & $10(26,3)$ & $2(5,26)$ & $8(21)$ & $7(18,4)$ & $2(5,26)$ \\
\hline
\end{tabular}

La péritonite et l'hypertrophie bénigne de la prostate (HBP) étaient les pathologies les plus fréquentes dans cette étude. La figure 1 nous montre la répartition des différentes indications chirurgicales.

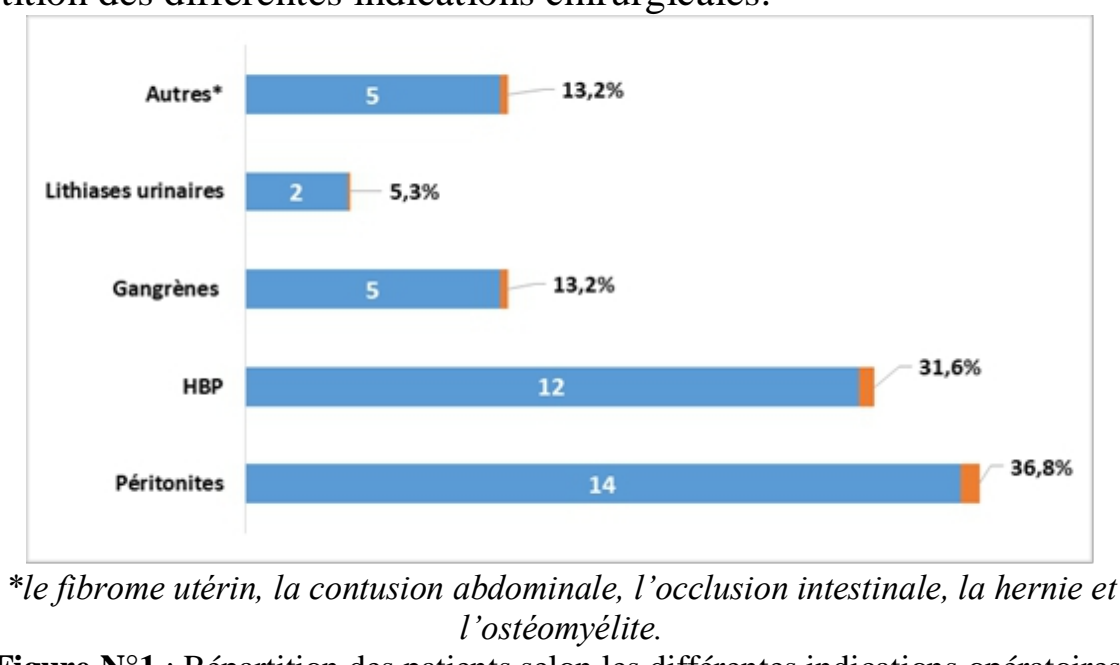

Figure $\mathbf{N}^{\circ} \mathbf{1}$ : Répartition des patients selon les différentes indications opératoires.

Les ISO étaient superficielles dans $36,8 \%(\mathrm{n}=14)$, profondes dans $42,1 \%$ $(\mathrm{n}=16)$ et d'organes dans $21,1 \%(\mathrm{n}=8)$. Les suppurations post opératoire avaient été observées entre le $2^{\text {ème }}$ et le $26^{\text {ème }}$ jour après l'intervention. Le délai 
médian était 6 jours. Ces suppurations avaient plus apparu chez les patients entre le $4^{\text {ème }}$ et $10^{\text {ème }}$ jour après l'intervention.

\section{Aspects bactériologiques}

Sur les 38 prélèvements, 33 cultures étaient positives soit $86,8 \%$ et $13,2 \%(n=5)$ étaient stériles.

Sur les 33 cas de culture positive, dix (10) espèces bactériennes ont été isolées. Les entérobactéries représentaient 69,7\% $(\mathrm{n}=23)$, les Cocci à Gram positif $24,2 \%(n=8)$ et les bacilles à Gram négatifs non-fermentaires $6,1 \%$ $(\mathrm{n}=2)$. Escherichia coli était l'espèce prédominante dans notre étude avec 45,5\% ( $n=15)$. Parmi ces 33 cultures positives, 45,5\% $(n=15)$ provenaient de la chirurgie digestive, $30,3 \%(n=10)$ de l'urologie et $24,2 \%(n=8)$ du service de traumatologie-orthopédie. Le Tableau II nous montre la répartition des différentes espèces bactériennes en fonction des différents services de spécialités chirurgicales.

Tableau II : Répartition des différentes espèces bactériennes

\begin{tabular}{|c|c|c|}
\hline Espèces bactériennes & Spécialités chirurgicales & Nombre (\%) \\
\hline \multicolumn{3}{|c|}{ Gram négatifs -Entérobactéries, $n=23(69,7)$} \\
\hline Escherichia coli & $\begin{array}{c}\text { CD }(\mathrm{n}=10), \text { TO }(\mathrm{n}=2), \text { Uro } \\
(\mathrm{n}=3)\end{array}$ & $15(45,5)$ \\
\hline Escherichia hermannii & $\mathrm{CD}(\mathrm{n}=1)$ & $1(3)$ \\
\hline Klebsiella pneumoniae & Uro $(n=4)$ & $4(12,1)$ \\
\hline Proteus vulgaris & TO $(n=1)$ & $1(3)$ \\
\hline Proteus mirabilis & Uro $(n=1)$ & $1(3)$ \\
\hline Enterobacter cloacae & Uro $(n=1)$ & $1(3)$ \\
\hline \multicolumn{3}{|c|}{ Gram négatifs- non fermentaires, $n=2(6,1)$} \\
\hline Pseudomonas aeruginosa & $\mathrm{CD}(\mathrm{n}=1), \mathrm{TO}(\mathrm{n}=1)$ & $2(6,1)$ \\
\hline \multicolumn{3}{|c|}{ Cocci Gram +, n= $8(24,2)$} \\
\hline Staphylococcus aureus & $\mathrm{CD}(\mathrm{n}=2), \mathrm{TO}(\mathrm{n}=3)$ & $6(18,2)$ \\
\hline Staphylococcus ssp & $\mathrm{CD}(\mathrm{n}=1), \mathrm{TO}(\mathrm{n}=1)$ & $1(3)$ \\
\hline Streptococcus du groupe $D$ & Uro $(n=1)$ & $1(3)$ \\
\hline Total & & $33(100)$ \\
\hline
\end{tabular}

$* C D=$ Chirurgie digestive, Uro $=$ Urologie, $T O=$ Traumatologie - orthopédie,

Pour les Cocci Gram positifs, les meilleures sensibilités étaient observées avec la vancomycine (100\%), du chloramphénicol (62,5\%) et l'érythromycine (57,1\%).Quant aux bacilles Gram négatifs, les meilleures sensibilités étaient données par l'imipenème (100\%), la colistine $(93,8 \%)$, l'aztréonam $(68,7 \%)$ et le chloramphénicol $(47,8 \%)$. Le tableau III nous montre la sensibilité des Cocci Gram positif et Cocci Gram négatif aux antibiotiques. 
La résistance des isolats d'entérobactéries était de $100 \%$ pour l'amoxicilline et le cotrimoxazole, 95,5\% pour l'association amoxicillineacide clavulanique, et $84,4 \%$ pour la céftriaxone.

\section{Discussion}

Les résultats de cette étude peuvent être discutés aussi avec les données de littérature sur les ISO. Les ISO sont des infections nosocomiales qui ont des conséquences graves en termes de morbidité et de mortalité et sont génératrices de surcoûts et de la compromission du pronostic (Allegranzi, 2016; Ouédraogo, 2011; Adrien, 2016; Simon, 2007; Ndayisaba, 1992; Wilson, 2004). Ces contraintes et difficultés décrites dans cette étude perturbent et majorent le risque d'infections nosocomiales pour les patients (Baker, 2016; Simon, 2007; Ndayisaba, 1992; Ngatchoukpo, 2014).

Dans les pays développés, les ISO affectent entre $0,7 \%$ et $5 \%$ des patients opérés (Fournel, 2017; Baker, 2016; Sisay, 2019; Sanger, 2016]. Dans notre contexte et dans la plupart des études en Afrique subsaharienne, le taux d'ISO est plus élevé et peut atteindre plus de 40\% en fonction de type de chirurgie (Ndayisaba, 1992; Adamou, 2017; Nejad, 2011; Abalo, 2010). Notre taux d'incidence était de 7,83\%. Ce taux est supérieur aux taux observés par Abdoulaye et al. 2018 à Niamey, Karim et al [Karim et al. 2000] en Tunisie et Chadli et al. 2005 au Maroc avec respectivement un taux de taux 2,47\%, $3,53 \%$ et $5,2 \%$.

Tableau III : Profils de sensibilité des 33 isolats aux antibiotiques testés des ISO.

\begin{tabular}{|c|c|c|}
\hline Antibiotiques & $\begin{array}{c}\text { Sensibilité des Cocci Gram } \\
+\end{array}$ & $\begin{array}{c}\text { Sensibilité des Cocci } \\
\text { Gram - }\end{array}$ \\
\hline Ampicilline & $2 / 6(33,3 \%)$ & $0 / 23(0 \%)$ \\
\hline Amoxicilline & $0 / 1(0 \%)$ & $0 / 19(0 \%)$ \\
\hline Oxacilline & $3 / 7(42,9 \%)$ & - \\
\hline Amikacine & $4 / 8(50 \%)$ & - \\
\hline Kanamycine & $4 / 8(50 \%)$ & $1 / 10(10 \%)$ \\
\hline Vancomycine & $\mathbf{8 / 8 ( 1 0 0 \% )}$ & - \\
\hline Erythromycine & $\mathbf{4 / 7}(\mathbf{5 7 , 1 \%})$ & $3 / 8(37,5 \%)$ \\
\hline Tobramycine & $3 / 8(37,5 \%)$ & $1 / 10(10 \%)$ \\
\hline Tétracycline & $0 / 1(0 \%)$ & $8 / 24(33,3 \%)$ \\
\hline Gentamicine & - & $11 / 23(47,8 \%)$ \\
\hline Chloramphénicol & $\mathbf{5 / 8 ( 6 2 , 5 \% )}$ & $\mathbf{3 / 3 ( 1 0 0 \% )}$ \\
\hline Imipénène & $4 / 7(57,1 \%)$ & $\mathbf{1 1 / 1 6 ( 6 8 , 5 \% )}$ \\
\hline Aztréonam & $0 / 7(0 \%)$ & $0 / 1(0 \%)$ \\
\hline Ceftazidine & $0 / 4(0 \%)$ & $2 / 24(8,3 \%)$ \\
\hline Ceftriaxone & $3 / 8(37,5 \%)$ & $1 / 17(5,9 \%)$ \\
\hline Céfalotine & $1 / 4(25 \%)$ & $0 / 1(0 \%)$ \\
\hline Pipéracilline & $3 / 8(37,5 \%)$ & $\mathbf{1 5 / 1 6}(\mathbf{9 3 , 8 \%})$ \\
\hline Colistine & - & $1 / 22(4,5 \%)$ \\
\hline Cotrimoxazole & - & $0 / 21(0 \%)$ \\
\hline
\end{tabular}


L'incidence dans notre étude est proche de celles rapportées par Hodonou et al (Hodonou, 2016) au Bénin et Ndayisaba et al (Ndayisaba, 1992) au Burundi qui avaient rapporté respectivement $7,3 \%$ et $7 \%$. Elle est largement inférieure à celle rapportée au Burkina (Ouédraogo, 2011).

Cependant, il faut reconnaître que certaines comparaisons sont peu satisfaisantes, du fait du contexte d'exercice différent et de la variabilité méthodologique des études. La fréquence des ISO est liée à plusieurs facteurs de risques dont, l'environnement, la qualité des infrastructures, les équipements, les conditions d'exercice médical, le professionnalisme du personnel et aussi l'attitude des demandeurs des soins. Les principaux facteurs favorisants les ISO sont liées à l'âge, à l'urgence du geste, aux conditions d'exercice, aux facteurs environnementaux, à un état de santé souvent précaire, au type d'intervention ainsi que la contamination (Baker, 2016; Simon, 2007; Alfonso-Sanchez, 2017; Altemeier, 1968; Kanassoua, 2015). Le type chirurgie et le niveau de contamination sont des éléments importants influençant la survenue des infections du site opératoire. Dans notre série, la chirurgie contaminée et chirurgie sale représentaient $89,5 \%$ et l'intervention chirurgicale était faite en urgence dans 57,9\%. Des nombreuses études ont confirmé le risque élevé des ISO pour les classes contaminée et sale (Lecuire, 2008; Sanger, 2016; Abdoulaye, 2018; Ngatchoukpo, 2014; Sanger, 2016; Tékpa, 2017). Pour Abalo et al. (Abalo, 2010), il y avait plus d'ISO dans les classes contaminée et sale d'Altemeier $(p=0,008)$. Dans notre étude, $89 \%$ des patients appartenaient aux classes III et IV d'Altemeier.

Ces ISO avaient affecté surtout les services de chirurgie viscérale et d'urologie avec respectivement $38,6 \%$ et $34,2 \%$ des cas. Des valeurs relativement identiques soit $31,8 \%$ avaient été observées en chirurgie viscérale à Parakou (Adrien et al. 2016). Le fait que les services de chirurgie viscérale et d'urologie soient beaucoup plus touchés se justifie par la fréquence élevée des péritonites $(34,2 \%)$ qui correspondent à la classe IV d'Alte Meier et l'HBP (adénome prostatique hypertrophique bénigne) (31,6\%) qui sont les principales causes d'interventions respectivement en chirurgie $B$ et en urologie.

La prédominance masculine $(86,8 \%)$ retrouvée dans cette étude a été rapportée dans plusieurs études (Ouédraogo A-S et al. 2011; Chadli et al. 2005; Alfonso-Sanchez et al. 2017). En effet, certains facteurs de risques des infections postopératoires sont plus retrouvés chez l'homme (Accidents des voies publiques dus à la vitesse en circulation, combats à armes blanches, les accidents sur les chantiers routiers ou de construction), mais surtout la spécificité de la chirurgie urologique pour adénome de la prostate à cause de sa proximité avec l'arbre urinaire.

La culture des prélèvements bactériologique est revenue positive chez 33 patients $(86,8 \%)$, les entérobactéries Gram négatif représentaient plus de 3/4 
de cas $(75,8 \%)$ avec une prédominance d'Escherichia coli dans 15 cas $(45,5 \%)$. Baker et al (Baker, 2016) avaient rapporté un taux de cultures positives de $86 \%$ similaire à notre série. Le résultat de l'étude bactériologique retrouvé dans notre étude concorde avec d'autres études hospitalières ayant noté la prédominance d'Escherichia coli avec un taux allant de 30,1\% à 64,7\% (Ouédraogo, 2011; Adrien, 2016). Ce qui peut s'expliquer par la prédominance d'affections digestives dans notre série où les germes endogènes provenant du tube digestif infectent le site opératoire. Nos résultats diffèrent de ceux rapportés dans d'autres études où le S.aureus était plus isolé dans les prélèvements bactériologiques, suivis des entérobactéries Gram négatif en particulier E. coli (Baker, 2016; Sisay, 2019; Ndayisaba, 1992; Abdoulaye, 2018; Ngatchoukpo, 2014).

Les résultats de nos antibiogrammes avaient montré que les Cocci Gram positifs étaient sensibles à la vancomycine, au chloramphénicol et à l'érythromycine dans respectivement 100\%, 62,5\% et 57,1\%. Quant aux bacilles Gram négatif, les meilleures sensibilités étaient données par l'imipenème $(100 \%)$, la colistine $(93,8 \%)$, l'aztréonam $(68,7 \%)$. Nous avons observé une forte résistance des entérobactéries pour l'amoxicilline (100\%), le cotrimoxazole $(100 \%)$ pour l'association amoxicilline-acide clavulanique $(95,5 \%)$ et pour la ceftriaxone $(84,4 \%)$ qui sont les antibiotiques de choix utilisés couramment en pré, per et post-opératoire. Ces antibiotiques sont les plus utilisés dans notre contexte dans le cadre d'une antibiothérapie probabiliste. Ces résistances pourraient s'expliquer par l'utilisation trop fréquente de ces antibiotiques en thérapeutique et aussi la pratique de l'automédication à l'origine d'une pression de sélection de mutants résistants à l'intérieur de notre hôpital. Les infections par bactéries multirésistantes sont de plus en plus décrites, reflétant les faillites de l'hygiène hospitalière en Afrique subsaharienne et imposant un changement de paradigme dans la prescription d'antibioprophylaxie et d'antibiothérapie (Ouédraogo, 2011; Adrien, 2016; Simon, 2007; Ndayisaba, 1992; Abdoulaye, 2018; Ngatchoukpo, 2014; Nejad, 2011; Chadli, 2005; Tékpa, 2017). Cette résistance élevée des bactéries isolées par rapport à certaines familles d'antibiotiques confirme qu'il s'agit bien de germes hospitaliers. Elle doit faire changer complètement la politique non seulement d'antibioprophylaxie dans les services chirurgicaux de l'hôpital, mais également les protocoles d'antibiothérapie probabiliste. En France, chaque année, plus de 150000 patients souffrent d'infections liées aux bactéries multirésistantes avec plus de 12500 décès (Carlet et Coz, 2015). Selon le comité d'Experts (Carlet et Coz, 2015), quatre axes majeurs ont été identifiés pour parvenir à limiter l'émergence et la dissémination des résistances bactériennes : 
- Approfondir les recherches, en particulier de nouveaux produits luttant contre l'antibiorésistance

- Mieux suivre l'évolution globale du phénomène via des indicateurs normalisés et partagés

- Améliorer l'usage des antibiotiques.

- Accroître la sensibilisation des populations au bon usage des antibiotiques (Carlet et Coz, 2015).

Cette étude présente des limites. Elle a été réalisée sur une courte période et l'enregistrement des données n'a pas été exhaustif. D'autres patients n'ont pas pu être enregistrés dans cette base de données comme ISO, car ils étaient exclus du fait qu'ils n'ont pas eu de prélèvement de pus envoyé au laboratoire pour étude. Les autres difficultés sont entre autres, l'insuffisance de certains matériels, réactifs ou disques d'antibiotiques au niveau de l'unité de bactériologie. Aussi des séries d'arrêts de travail était observé par les syndicats ce qui a handicapé les activités au niveau des services de chirurgie et de laboratoire. Cependant les résultats obtenus sont encourageants. Ils permettront aux services hospitaliers d'avoir une idée sur leur écologie bactérienne, mettre en place leur politique de prévention des infections associées aux soins et d'améliorer la prescription des antibiotiques.

\section{Conclusion}

Cette étude a montré que les infections du site opératoires constituent un problème majeur dans notre hôpital. Escherichia coli et le staphylococcus aureus sont les bactéries les plus isolées respectivement en chirurgie digestive et traumato-orthopédique. Ces germes hospitaliers développent de plus en plus une multirésistance aux antibiotiques prescrits en première intention dans notre pratique. L'instauration d'une stratégie hospitalière préventive et l'utilisation rigoureuse d'antibiotiques s'avèrent urgentes et indispensables.

Remerciements : aux personnels des services de laboratoire, de chirurgie et d'infectiologie de l'Hôpital national de Zinder.

\section{References:}

1. Abalo, A., A. Walla, G. Ayouba, et al. (2010). Infection Du Site Opératoire En Chirurgie Orthopédique Dans Un Pays En Voie de Développement. Revue de Chirurgie Orthopédique et Traumatologique 96(1): 112-117.

2. Abdoulaye, O, Harouna Amadou ML, Oumarou A, et al. (2018). Aspects Épidémiologiques et Bactériologiques Des Infections Du Site Opératoire (ISO) Dans Les Services de Chirurgie à l'Hôpital National de Niamey (HNN). The Pan African Medical Journal 31. https://www.ncbi.nlm.nih.gov/pmc/articles/PMC6430836/ 
3. Adamou, H., O. Habou, I. Amadou-Magagi, et al. (2017). Les Péritonites Aiguës Non Traumatiques de l'enfant: Étiologies et Pronostic Chez 226 Patients à l'hôpital National de Zinder, Niger. Méd et Santé Trop 27(3): 264-269.

4. Adrien, Hodonou Montcho, Hounkponou Fany, Allodé Salako Alexandre, et al. (2016). Aspects Bacteriologiques Des Infections Du Site Operatoire Au Centre Hospitalier Departemental Du Borgou A Parakou (Benin). European Scientific Journal, ESJ 12(9):353-60.

5. Alfonso-Sanchez, Jose L., Isabel M. Martinez, Jose M. MartínMoreno, Ricardo S. González, and Francisco Botía (2017). Analyzing the Risk Factors Influencing Surgical Site Infections: The Site of Environmental Factors. Canadian Journal of Surgery 60(3): 155-161.

6. Allegranzi, Benedetta, Peter Bischoff, Stijn de Jonge, et al. (2016). New WHO Recommendations on Preoperative Measures for Surgical Site Infection Prevention: An Evidence-Based Global Perspective. The Lancet Infectious Diseases 16(12): e276-e287.

7. Altemeier, W. A., W. R. Culbertson, and R. P. Hummel (1968). Surgical Considerations of Endogenous Infections--Sources, Types, and Methods of Control. The Surgical Clinics of North America 48(1): 227-240.

8. Baker, Arthur W., Kristen V. Dicks, Michael J. Durkin, et al. (2016). Epidemiology of Surgical Site Infection in a Community Hospital Network. Infection Control and Hospital Epidemiology 37(5): 519526.

9. Carlet J Et Coz P. (2015). Rapport du groupe de travail special pour la preservation des antibiotiques. https://epidmeteo.fr/france/antibio-resistance. 150 pages.

10. Chadli, M., N. Rtabi, S. Alkandry, et al. (2005). Incidence Des Infections Du Site Opératoire Étude Prospective à l'hôpital Militaire d'instruction Mohamed-V de Rabat, Maroc. Médecine et Maladies Infectieuses 35(4): 218-222.

11. Fournel, Ludovic. (2017). Les Infections Du Site Opératoire. Revue Francophone de Cicatrisation 1(2): 27-30.

12. Horan, Teresa C., Mary Andrus, and Margaret A. Dudeck (2008). CDC/NHSN Surveillance Definition of Health Care-Associated Infection and Criteria for Specific Types of Infections in the Acute Care Setting. American Journal of Infection Control 36(5): 309-332.

13. Kanassoua, K., I. Kassegne, E. Sakiye, Et Al. (2015). Infections Du Site Opératoire En Chirurgie Générale Dans Un Hopital Regional Au Togo. Rev Cames Santé 3: 2424-7243.

14. Karim. H, Kacem. C, Kolsi K et al. (2000). Facteurs de Risque de l'infection Des Plaies Opératoires En Chirurgie Digestive. Etude 
Rétrospective Sur 3000 Plaies Opératoires. Tunisie Médicale 78(11): 634-640.

15. Ndayisaba, G., L. Bazira, G. Gahongano, A. Hitimana, and R. Arayuba (1992). Bilan Des Complications Infectieuses En Chirurgie Générale. Analyse d'une Série de 2218 Interventions. Médecine d'Afrique Noire 39: 571-73.

16. Nejad. SB, Allegranzi B, Shamsuzzoha. BS, Ellis. B, and Pittet. D (2011). Health-Care-Associated Infection in Africa: A Systematic Review. Bulletin of the World Health Organization 89: 757-765.

17. Ngaroua. J, Eloundou Ngah. J, Bénet. T, and Djibrilla Y. (2016). Incidence Des Infections Du Site Opératoire En Afrique SubSaharienne: Revue Systématique et Méta-Analyse. Pan African Medical Journal

24(1). https://www.ajol.info/index.php/pamj/article/view/144078 .

18. Gaudeuille A, Ngatchoukpo VN. (2014). Complications post opératoires dans le service de chirurgie pediatrique de Bangui. Revue Africaine et Malgache de Recherche Scientifique/Sciences de La Santé 2(1):4-8.

19. Ouédraogo A-S., Somé DA., Dakouré PWH., Sanon BG., Birba E., Poda GEA., Kambou T., (2011). Profil bactériologique des infections du site opératoire au centre hospitalier universitaire Souro Sanou de Bobo Dioulasso. Med Trop, 71 : 49-52.,

20. Sanger, Patrick C, Gabrielle H van Ramshorst, Ezgi Mercan, et al. (2016). A Prognostic Model of Surgical Site Infection Using Daily Clinical Wound Assessment. Journal of the American College of Surgeons 223(2): 259-270.e2.

21. Simon, F., P. Kraemer, J. J. De Pina, E. Demortiere, and C. Rapp (2007) Le Risque Nosocomial En Afrique Intertropicale-Partie 2: Les Infections Des Patients. Med Trop 67(2): 197-20.

22. Sisay, Mekonnen, Teshager Worku, and Dumessa Edessa. (2019). Microbial Epidemiology and Antimicrobial Resistance Patterns of Wound Infection in Ethiopia: A Meta-Analysis of Laboratory-Based Cross-Sectional Studies. BMC Pharmacology \& Toxicology 20(1): 35.

23. Tékpa, B. J. D., G. Tékpa, P. A. I. Mapouka, et al. (2017). La Prévention Des Infections Du Site Opératoire En Orthopédie Dans Un Pays En Voie de Développement. Revue de Chirurgie Orthopédique et Traumatologique 103(7): 823-827.

24. Wilson, A P R, C Gibbons, B C Reeves, et al. (2004). Surgical Wound Infection as a Performance Indicator: Agreement of Common Definitions of Wound Infection in 4773 Patients. BMJ : British Medical Journal 329(7468): 720. 